\title{
A linear electrooptic effect in silicon, induced by use of strain
}

Fage-Pedersen, Jacob; Frandsen, Lars Hagedorn; Lavrinenko, Andrei; Borel, Peter Ingo

Published in:

2006 3rd IEEE/LEOS International Conference on Group IV Photonics - Proceedings CD-ROM.

Link to article, DOI:

10.1109/GROUP4.2006.1708157

Publication date:

2006

Document Version

Publisher's PDF, also known as Version of record

Link back to DTU Orbit

Citation (APA):

Fage-Pedersen, J., Frandsen, L. H., Lavrinenko, A., \& Borel, P. I. (2006). A linear electrooptic effect in silicon, induced by use of strain. In $20063 \mathrm{rd}$ IEEE/LEOS International Conference on Group IV Photonics - Proceedings CD-ROM. (pp. 37-39). IEEE/LEOS. https://doi.org/10.1109/GROUP4.2006.1708157

\section{General rights}

Copyright and moral rights for the publications made accessible in the public portal are retained by the authors and/or other copyright owners and it is a condition of accessing publications that users recognise and abide by the legal requirements associated with these rights.

- Users may download and print one copy of any publication from the public portal for the purpose of private study or research.

- You may not further distribute the material or use it for any profit-making activity or commercial gain

- You may freely distribute the URL identifying the publication in the public portal 


\title{
A linear electrooptic effect in silicon, induced by use of strain
}

\author{
Jacob Fage-Pedersen, Lars H. Frandsen, Andrei V. Lavrinenko, and Peter I. Borel
}

\author{
$\mathrm{COM} \cdot \mathrm{DTU}$, Department of Communications, Optics \& Materials, Nano•DTU, Technical University of Denmark, \\ building 345V, DK-2800 Kgs. Lyngby, Denmark, fage@ com.dtu.dk
}

\begin{abstract}
The crystal structure in a silicon waveguide can be distorted by application of strain. Thereby, the otherwise forbidden linear electrooptic (Pockels) effect is induced, opening a new route for optical modulation in silicon.
\end{abstract}

\section{Introduction}

Silicon photonics is undergoing a spectacular development these years, driven by short-reach interconnection demands (from several $\mu \mathrm{m}$ to a few $\mathrm{m}$ ) and the pushing need for low-cost electronicphotonic integration at high bit rates. In these systems, the electrooptic modulator is a key component. Most commercial modulators, such as those made in $\mathrm{LiNbO}_{3}$, make use of the linear electrooptic (Pockels) effect. However, this effect is intrinsically zero in single-crystalline Si due to the inversionsymmetry of the crystal structure [1]. We demonstrate [2] that a straining layer can be employed to efficiently break the crystal symmetry in a silicon waveguide, such that a finite Pockels coefficient is generated. This method represents a new method for making electrooptic modulation in Si. Whereas the far majority of proposed silicon modulators $[3,4]$ use the plasma-dispersion effect, the Pockels effect offers advantages of requiring no change of carrier concentration and no current flow for modulation. Therefore it is expected to have very low power consumption and an operation speed which is limited primarily by electrode design.

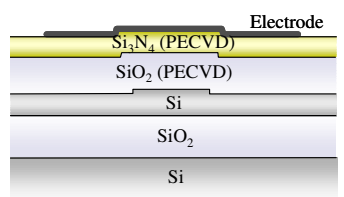

(a)

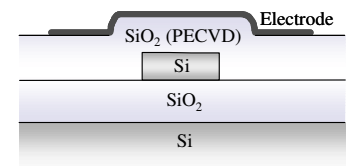

(b)

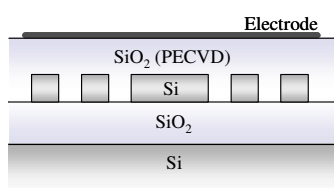

(c)

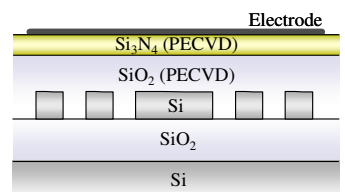

(d)

Figure 1. Cross-sectional schematics of waveguides with glass cladding: (a) Shallowly etched ridge, (b) photonic wire, (c) and (d) PCWs. The PECVD glass layers exert stress on the Si waveguide layer. The PECVD glass only partially fills the holes of the PCWs.

\section{Method}

Different batches of waveguides were investigated. They are shown with cross-sectional schematics in Fig. 1. The waveguides were fabricated in silicon-on-insulator (SOI) having a 1 or 1.2- $\mu \mathrm{m}$ thick buried oxide and a Si device layer with a thickness between 220 and $340 \mathrm{~nm}$. The substrate and device layers are $p$-type with resistivities $\sim 15$ and $10 \Omega$-cm, respectively (Fig. 1[a,d]), or $\sim 30$ and $30 \Omega$-cm, respectively $(1[\mathrm{~b}, \mathrm{c}])$. Photonic wires of width $430 \mathrm{~nm}$ and photonic-crystal waveguides (PCWs) were defined with either deep-UV or electron-beam lithography, and plasma etched. On all batches, a top cladding layer of silica glass $\left(\mathrm{SiO}_{2}\right)$ with a thickness between 1.2 and $1.5 \mu \mathrm{m}$ was deposited with plasma-enhanced chemical vapour deposition (PECVD). The strain of this layer can be made either compressive or tensile, with a typical value around $\sim 0.3 \mathrm{GPa}$. In two batches, a $0.75-\mu \mathrm{m}$ thick layer of silicon nitride glass (approximate stoichiometry: $\mathrm{Si}_{3} \mathrm{~N}_{4}$ ) with a high, compressive strain of $\sim 1 \mathrm{GPa}$ was also deposited. Finally, metal electrodes were formed on the top surface. Electrooptic (EO) measurements were performed by applying an a.c. modulation voltage to the top electrode, keeping the Si substrate at ground potential. While modulating, the optical transmission was monitored either through an on-chip MachZehnder interferometer (MZI) having an electrode over one of the arms, or through a fibre-based MZI 
setup with a waveguide placed in one arm and a calibrated $\mathrm{LiNbO}_{3}$ modulator in the other arm, balancing the modulation of the two arms. The measurements were made with transverse-electric (TE) polarisation at modulation frequencies between 70 and $500 \mathrm{kHz}$, avoiding thermal effects that are generally observed below $\sim 10 \mathrm{kHz}$.

\section{Results and analysis}

For a ridge waveguide with very shallow etch $(\sim 30 \mathrm{~nm})$ and PECVD upper cladding glasses, as shown in Fig. 1(a), the EO nonlinearity is below the detection limit. This is so even though a straining layer of $\mathrm{Si}_{3} \mathrm{~N}_{4}$ has been applied on top. In contrast, a finite nonlinearity is found in waveguides that have been etched through the whole silicon layer and covered with a straining PECVD oxide, shown in Figs. 1(b) and 1(c). These observations suggest that the silicon waveguide core must be allowed to deform to the sides (the waveguide must be fully etched) in order to achieve a breaking of the crystal symmetry sufficient to induce a measurable Pockels effect. In the structure of Fig. 1(d), a $\mathrm{Si}_{3} \mathrm{~N}_{4}$ straining layer has been deposited on top of the upper silica cladding. This structure exhibits a dramatically enhanced nonlinearity compared to the structures of Figs. 1(b) and 1(c), even though the optical field has no overlap with the $\mathrm{Si}_{3} \mathrm{~N}_{4}$ layer. This demonstrates that the cause of the nonlinearity is the large strain field, induced by the $\mathrm{Si}_{3} \mathrm{~N}_{4}$ layer and acting on the silicon waveguide through the silica cladding: With tensile strained $\mathrm{SiO}_{2}$, the second-order nonlinear susceptibility $\chi^{(2)}$ is of the order $\sim 0, \sim 1$, and $\sim 15 \mathrm{pm} / \mathrm{V}$ for the configurations of Fig. 1(a), 1(c), and 1(d), respectively. With compressively strained $\mathrm{SiO}_{2}, \chi^{(2)} \sim 5-10$ $\mathrm{pm} / \mathrm{V}$ for configurations $1(\mathrm{~b})$ and $1(\mathrm{c})$.

For ease of comparison, these $\chi^{(2)}$ values were obtained after scaling linearly down from the values obtained at the actual group index $n_{\mathrm{g}}$ of the particular waveguide, to those expected at the bulk index, assumed for simplicity to be 3.5. For the highest strain, the electrode length $L$ times the electrical bias $V_{\pi}$ required for a phase shift of $\pi$ is a couple of hundred $V-\mathrm{cm}$, which should be improved by at least an order of magnitude for practical exploitation. However, the values reported here are first results, and large improvement is expected with refined waveguide design, optimised strain, and reduced electrode distance, possibly in combination with utilisation of slow light, as shown further below.

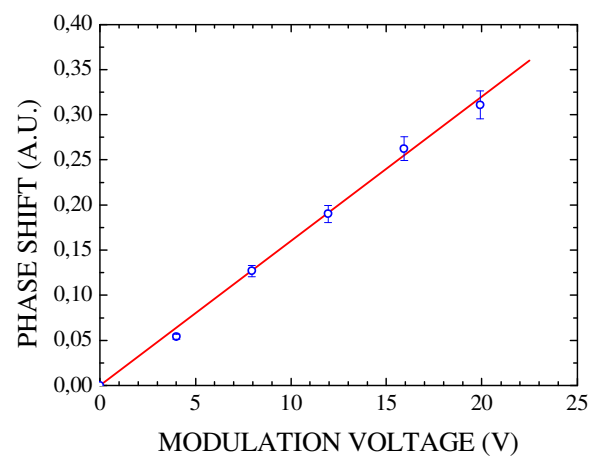

Figure 2. Measured phase shift (arbitrary units) as a function of modulation voltage, measured with 70-kHz. modulation at a wavelength of $1530 \mathrm{~nm}$. The straight line is a linear fit.

Fig. 2 demonstrates that the measured phase shift is directly proportional to the applied modulation voltage $(4-20 \mathrm{~V})$. This corroborates that the effect is indeed a linear electrooptic (Pockels) effect. No alternative effect was found to be a likely cause of the optical modulation, as discussed in ref. [2]. In particular, the effect is not related to the presence of charge carriers since the a.c. modulation is unaffected by a simultaneous application of a high d.c. voltage that depletes the structure efficiently.

Since the strain-induced nonlinearity is easily induced in a PCW, one can take advantage of slowlight enhancement of the light-matter interaction. In Fig. 3(a) we show the output spectrum of an on-chip MZI with a PCW in one arm, covered by an electrode. The rapidly increasing oscillations are a clear sign 
of an increasing group index in the PCW when approaching the cut-off at $1564 \mathrm{~nm}$ of the in-gap guided mode [5]. Fig. 3(b) shows that the measured phase shift in the PCW for a given modulation voltage (i.e., the nonlinearity) is enhanced with increasing $n_{\mathrm{g}}$, being approximately proportional to $n_{\mathrm{g}}$ up to at least $n_{\mathrm{g}} \approx$ 35. In ref [2] we demonstrate phase-shift enhancements with $n_{\mathrm{g}}$ values up to a couple of hundred.
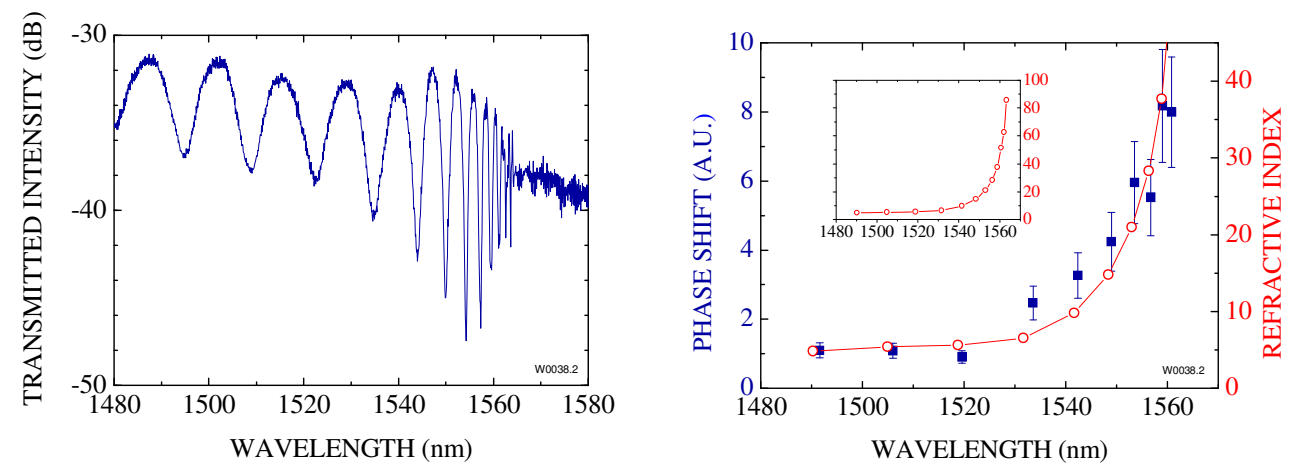

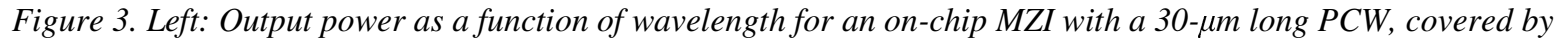
an electrode, in one arm (device cross section as in Fig. 1[b, c]). Right: Measured phase shift (-) and effective group index( ) in the PCW.

Let us finally point out that a $\chi^{(2)}$ nonlinearity arising from distortion of a Si crystal under a straining $\mathrm{SiO}_{2}$ layer has previously been evidenced by second-harmonic generation (SHG) [6,7]. We believe that the present investigations, performed in waveguides, are the first ones that point to an EO strain-induced $\chi^{(2)}$. In contrast to the SHG experiments, we were not able to detect the EO effect in those samples where the silicon layer is almost flat (Fig. 1[a]). Let us also note that for traditional Si waveguides, having crosssectional dimensions of several $\mu \mathrm{m}$, we expect that the strain effect will be rather small. The reason is that for large waveguides, much less lattice deformation is expected in the centre region where the optical field is maximum.

\section{Conclusion}

Distortion of the crystal symmetry is an attractive new route to enable optical modulation in Si. A Pockels effect can be induced by straining the waveguide core by application of a highly straining layer (e.g. $\mathrm{Si}_{3} \mathrm{~N}_{4}$ ). The method has high potential due to the expected low power consumption and ultra-high speed operation that is foreseen, and due to the use of standard CMOS processes in the fabrication. Furthermore, the method can be exploited in a slow-light PCW for increased, effective nonlinearity.

\section{References}

[1] Robert W. Boyd, Nonlinear Optics, second edition (Academic Press, 2003), Chap. 1.

[2] Rune S. Jacobsen, Karin Andersen, Peter I. Borel, Jacob Fage-Pedersen, Lars H. Frandsen, Ole Hansen, Martin Kristensen, Andrei V. Lavrinenko, Gaid Moulin, Haiyan Ou, Christophe Peucheret, Beáta Zsigri, and Anders Bjarklev: "Strained silicon as a new electro-optic material", Nature 441, 199 (2006).

[3] Ling Liao, Dean Samara-Rubio, Michael Morse, Ansheng Liu, Dexter Hodge, Doron Rubin, Ulrich D. Keil, and Thorkild Franck: "High speed silicon Mach-Zehnder modulator", Opt. Express 13, 3129 (2005)

[4] Qianfan Xu, Bradley Schmidt, Sameer Pradhan, and Michal Lipson: "Micrometre-scale silicon electro-optic modulator", Nature 435, 325 (2005)

[5] Rune Jacobsen, Andrei Lavrinenko, Lars Frandsen, Christophe Peucheret, Beata Zsigri, Gaid Moulin, Jacob Fage-Pedersen, and Peter Borel: "Direct experimental and numerical determination of extremely high group indices in photonic crystal waveguides", Opt. Express 13, 7861 (2005)

[6] Jung Y. Huang, "Probing Inhomogeneous Lattice Deformation at Interface of $\mathrm{Si}(111) / \mathrm{SiO}_{2}$ by Optical SecondHarmonic Reflection and Raman Spectroscopy": Jpn. J. Appl. Phys 33, 3378 (1994)

[7] W. Daum, H.-J. Krause, U. Reichel, and H. Ibach: "Identification of Strained Silicon Layers at $\mathrm{Si}_{-} \mathrm{SiO}_{2}$ Interfaces and Clean Si Surfaces by Nonlinear Optical Spectroscopy", Phys. Rev., Lett. 71, 1234 (1993). 